\title{
CONSTRUÇÃO DE ESTRATÉGIAS DE ATENÇÃO EM SAÚDE PARA DIAGNÓSTICO DE GONORREIA E CLAMÍDIA COMO PROPOSTA DE ATIVIDADE EXTENSIONISTA ${ }^{1}$
}

\author{
BUILDING OF HEALTHCARE STRATEGIES FOR THE DIAGNOSIS OF \\ GONORRHEA AND CHLAMYDIA AS A PROPOSAL FOR EXTENSION ACTIVITY
}

\author{
Luisa Donato Bortoluzzi ${ }^{2}$, Ana Luiza Anversa Antonello², Wanessa de Carvalho Vieira ${ }^{2}$, \\ Gabriela Heloiza Cadore², Márcia Gabriela Rodrigues de Lima ${ }^{3}$, Raquel Soares Moreira ${ }^{4}$, \\ Huander Felipe Andreolla ${ }^{5}$ e Jane Beatriz Limberger ${ }^{6}$
}

\section{RESUMO}

O planejamento e a implementação de estratégias de atenção à saúde são imprescindíveis para o aprimoramento das políticas públicas, pois permitem a ampliação das ações desenvolvidas e efetivação de seus objetivos. A integração entre ensino, serviço e comunidade agrega forças e conhecimentos para este planejamento, resultando em benefícios para todos os atores envolvidos. O presente estudo busca relatar a experiência de atividades de uma extensão universitária sobre a construção ferramentas de organização dos serviços para diagnóstico gonorreia e clamídia desenvolvidas por estudantes do curso de Biomedicina, residentes da Residência Multiprofissional em Atenção Clínica Especializada com Ênfase em Infectologia e Neurologia e servidores do Serviço de Assistência Especializada (SAE) e Centro de Testagem e Aconselhamento (CTA) para infecções sexualmente transmissíveis (ISTs) da cidade de Santa Maria, RS. Esta extensão foi realizada no período de setembro a novembro de 2020, utilizando como ferramenta metodológica a "espiral construtivista", aliada reuniões de discussão e avaliação. Como resultados, destaca-se a produção do fluxograma de atendimento, um manual de procedimentos para os servidores e materiais de educação em saúde para os usuários do serviço. Com isso, evidenciou-se a importância dessa atividade, pois, através da integração dos diferentes atores se produziu novas metodologias de educação em saúde, organização do serviço e ampliação do conhecimento sobre o assunto, qualificando o processo de formação dos estudantes.

Palavras-chave: Extensão Comunitária, Infecções Sexualmente Transmissíveis, Educação em Saúde.

\section{ABSTRACT}

The planning and implementation of health care strategies are essential for the improvement of public policies, as they allow for the expansion of developed actions and the realization of their goals. The integration between teaching, service and community adds strength and knowledge to this planning, resulting in benefits for all actors involved. This study seeks to report the experience of activities of a university extension on the construction of tools for the organization of services for the diagnosis of gonorrhea and chlamydia, developed

1 Trabalho de Extensão Universitária.

2 Acadêmicas do curso de Biomedicina, Universidade Franciscana, Santa Maria, RS. E-mails: luisad.bortoluzzi@gmail.com; a.luiza-ant@hotmail.com; wanessacvieira@hotmail.com; gabrielacadore@hotmail.com

3 E-mail: grlmarcia@yahoo.com.br

4 E-mail: raquels.moreira@hotmail.com

5 Professor dos cursos de Biomedicina e Medicina, Universidade Franciscana, Santa Maria, RS. E-mail: huander.andreolla@ ufn.edu.br

6 Professora dos cursos de Farmácia e Biomedicina, Universidade Franciscana, Santa Maria, RS. E-mail: janebeatriz@ ufn.edu.br 
by students of the Biomedicine course, residents of the Multiprofessional Residency in Specialized Clinical Care with Emphasis on Infectology and Neurology, and servers of the Specialized Assistance Service (SAE) and Testing and Counseling Center (CTA) for ISTs in the city of Santa Maria, RS. This extension was carried out from September to November 2020, using the "constructivist spiral" as a methodological tool, together with discussion and evaluation meetings. As a result, there is the production of the service flowchart, a manual of procedures for servers and health education materials for service users. With that, the importance of this activity was evidenced, because, through the integration of the different actors, new methodologies of health education were produced, organization of the service and expansion of knowledge on the subject, qualifying the process of formation of the students.

Keywords: Community-Institutional Relations, Sexually Transmitted Diseases, Health Education.

\section{INTRODUÇÃO}

A realidade social brasileira é caracterizada por apresentar profundas desigualdades socioeconômicas, herança do seu passado colonial e derivada da industrialização de alguns segmentos estratégicos, o que resulta em escassa redistribuição social. Este cenário se reflete nas políticas públicas de saúde, e apesar das profundas mudanças implementadas nos últimos 30 anos com a reforma sanitária brasileira e a criação do SUS, ainda há um longo caminho a ser percorrido para a garantia da equidade, universalidade e integralidade no acesso à saúde (MACHADO; LIMA; BAPTISTA, 2017).

Entende-se por política pública o conjunto de intervenções planejadas do poder público com a finalidade de resolver situações sociais problemáticas (GIOVANNI; NOGUEIRA, 2018). Já as políticas públicas de saúde estão inseridas em um contexto multidisciplinar, e devem contemplar a participação de diferentes atores da sociedade na gestão dos serviços de saúde, promovendo um modelo de articulação e cooperação entre os envolvidos em prol de objetivos em comum (BEZERRA, 2019).

Sendo a equidade uma das mais importantes diretrizes do SUS, há que se considerar sua importância na elaboração de políticas públicas de saúde, contemplando o entendimento de que existem necessidades especiais de saúde para grupos específicos, e promover a realização de práticas que reconheçam estas diferenças é fundamental para a concretização do acesso (DIAS et al., 2019). No cenário de atenção, a promoção da saúde e a prevenção de doenças no âmbito da saúde sexual e reprodutiva são de considerável importância em função do alto custo das tecnologias em saúde envolvidas, da escassez de recursos públicos e de seu impacto na morbimortalidade da população em risco (PAIVA; CAETANO, 2020).

Apesar das ações de saúde sexual e reprodutiva estarem previstas em diversas políticas e programas e serem um dos eixos prioritários da atenção primária me saúde, não há uma efetiva implementação nos serviços, permanecendo uma lacuna entre as políticas e as práticas organizacionais e sociais (BRASIL, 1985; 1996; 2008; 2016; 2018). A busca de soluções perpassa a participação de todos os atores envolvidos, e no cenário municipal, contempla os trabalhadores em saúde, a gestão, 
os usuários e pode contar com a colaboração de instituições de ensino superior, por meio de atividades de cunho extensionista.

Nesse contexto, a extensão universitária se apresenta como uma oportunidade de aproximação da academia com a comunidade, em um conjunto de atividades direcionadas para o bem-estar físico e social dos participantes, a partir do levantamento de suas demandas. É possível desta forma associar ensino e pesquisa, produzindo novos conhecimentos e transformado a realidade social, além de promover a melhoria da qualidade de vida dos indivíduos e possibilitar aos acadêmicos novos conhecimentos a partir da troca de saberes (FORPROEX, 2006).

Deste modo, este trabalho tem o propósito de relatar a experiência vivenciada por acadêmicos do Curso de Biomedicina em uma atividade de planejamento de ações extensionistas da disciplina de Políticas de Saúde, tendo como cenário um centro de atenção especializada no atendimento de pessoas com infecções sexualmente transmissíveis. A ação teve por objetivos (1) sistematizar um fluxograma de atendimento para diagnóstico de clamídia e gonorreia (2) elaborar um manual para os trabalhadores em saúde com as orientações para realização dos respectivos exames e (3) construir folhetos informativos para os usuários do serviço, com foco na prevenção de Infecções Sexualmente Transmissíveis (ISTs), contando com a participação dos atores envolvidos no processo.

\section{MATERIAIS E MÉTODOS}

Este artigo se constitui em um relato de experiência que descreve aspectos vivenciados pelos autores, na oportunidade de acompanhamento da Disciplina de Políticas de Saúde, componente curricular do curso de Biomedicina. Adotaram-se como estratégias metodológicas: o aprofundamento teórico, identificação de problemas, formulação de explicações, elaboração de questões, busca de novas informações, construção de novos significados e avaliação dos processos e produtos, conforme proposto por Lima (2016) para a construção da espiral construtivista, referencial que norteia as atividades extensionistas da disciplina.

A revisão bibliográfica foi realizada nas plataformas de bancos de dados de informações técnicas cientificas, a saber: Scielo, Pubmed e em documentos oficiais da secretaria de saúde municipal. Foram pesquisadas referências que contemplassem os seguintes temas: políticas públicas; infecções sexualmente transmissíveis; ações integradas; educação para a saúde, intesetorialidade, integralidade, vigilância, promoção da saúde, planejamento em saúde. Estes artigos foram lidos e sintetizados para a estruturação do referencial teórico e sustentação da discussão.

A identificação dos problemas aconteceu por meio da observação de campo, durante o mês de setembro de 2020, por meio de reuniões de discussão entre as estudantes, residentes em Atenção Clínica Especializada com Ênfase em Infectologia e Neurologia e a equipe de saúde do Serviço de Assistência Especializada (SAE) e Centro de Testagem e Aconselhamento (CTA) para ISTs do município. 
A partir do elenco de problemas, se seguiram as demais etapas integrantes da espiral construtivista, que resultou na elaboração do fluxograma, manual de procedimentos e panfletos informativos sobre clamídia e gonorreia.

\section{RESULTADOS E DISCUSSÃO}

\section{Aprofundamento teórico}

O primeiro resultado foi a contextualização e compreensão, por parte do grupo de extensionistas, do papel das políticas públicas de saúde na assistência e prevenção de doenças no âmbito da saúde sexual e reprodutiva, bem como sua interface com outras políticas de saúde intersetoriais e o papel da vigilância em saúde na elaboração do planejamento de ações de interesse.

A sexualidade é uma característica inerente ao ser humano e por isso, é uma dimensão inseparável do existir. Portanto, a educação sexual, implícita ou explicitamente, está intimamente ligada à dimensão cultural e sociopolítica do indivíduo (FONSECA, 2002). Atividades educativas contribuem para a promoção da saúde e prevenção de infecções sexualmente transmissíveis, tornando a escola um espaço intersetorial potente para a aprendizagem. Contudo, percebe-se que o processo de educação em saúde é prejudicado pela não-percepção das falhas existentes na educação preventiva pelos profissionais da saúde ou outros espaços em que ela ocorre. Observa-se também a necessidade da participação de múltiplos setores na educação sexual, o que inclui a participação de instituições de ensino superior, especialmente vinculadas aos cursos da área da saúde (HIGA et al., 2015).

É preciso ponderar que uma proposta educativa não deve considerar apenas o modelo biomédico de tratamento de doenças mas que abranja questões relativas ao gênero, à sexualidade, à autonomia e à liberdade para a construção de práticas não discriminatórias que garantam a promoção, a proteção e o exercício da sexualidade e da reprodução como um direito. Deve-se assim pautar as atividades de educação em saúde na integralidade da assistência à saúde, em diferentes níveis de cuidado (LEMOS, 2014). Contudo, o modo como as instituições e as práticas de saúde se organizam, resulta em uma atenção fragmentada e de baixa resolutividade, com o cuidado dirigido a queixas clínicas e ainda muito direcionadas ao público feminino e/ou adolescente (TELO; WITT, 2018).

Em relação ao cuidado às pessoas com IST, este deve ser pautado na melhor evidência científica disponível, associada à fatores contextuais como a experiência do profissional, as características individuais e a potencialidade do sistema de saúde. Ao planejarmos a organização de um fluxo de atendimento para o diagnóstico e tratamento das pessoas com IST, deve-se considerar que as recomendações estejam adequadamente sistematizadas e acessíveis aos profissionais de saúde, que por sua vez devem ter a disposição condições de trabalho adequadas e capacitação para o desenvolvimento das atividades de cuidado (GALVÃO; COSTA; GARCIA, 2021). 
Já no contexto da vigilância em saúde, destaca-se a importância da efetiva manutenção das notificações de infecções e doenças sexualmente transmissíveis que permitem o monitoramento de fatores de risco e proteção para a população e fomento de políticas públicas e intervenções que sejam direcionadas à população sexualmente ativa, respeitando suas particularidades e subjetividades (FELISBINO-MENDES et al., 2018). Para tanto, é necessária a estruturação de um sistema de indicadores para diagnóstico, monitoramento e investigação das questões relacionadas à Saúde Sexual e Reprodutiva, abarcados em uma Dimensão contextual, aferido por meio de indicadores como população, raça, sexo e grupos etários, escolaridade, nível de desenvolvimento socioeconômico (IDH); temáticas relativas às condições de saúde tais como esperança de vida, mortalidade infantil; e as temáticas relativas às condições de vida como perfil ocupacional e rendimentos. Já a Dimensão da saúde sexual e reprodutiva diz respeito a questões mais específicas, como gravidez, ISTs e outras doenças do aparelho genital, gestação e puerpério, além de políticas públicas específicas (CORREA; ALVES; JANNUZZI, 2015).

\section{Identificação de problemas}

Para a concretização da atividade extensionista, inicialmente foi agendado um encontro com a psicóloga do Serviço de Assistência Especializada (SAE) e Centro de Testagem e Aconselhamento (CTA) para ISTs, a fim de discutir as prioridades de intervenção para o serviço. Durante a reunião, foi informado que os pacientes que são indicados à Profilaxia Pré-Exposição (PrEP) atualmente realizam os seguintes exames: estudo laboratorial de doenças venéreas (VDRL), detecção qualitativa de anticorpos contra o antígeno de superfície do vírus da Hepatite B (anti-Hbs), exame qualitativo de urina (EQU), ureia, creatinina sérica, aspartato aminotransferase/alanina aminotransferase (ALT/AST) e anticorpos totais contra o vírus da hepatite A (anti-HVA total), os quais são realizados em parceria com o laboratório do município.

Diante disso, percebeu-se a necessidade de implantar também o diagnóstico de Chlamydia trachomatis e Neisseria gonorrohoreae os quais não são realizados no laboratório parceiro e seria uma oportunidade de colaboração do Laboratório de Biologia Molecular da Universidade Franciscana.

\section{Formulação de explicações}

Devido ao grande número de indivíduos portadores de IST's na cidade de Santa Maria, torna-se necessário um diagnóstico rápido destas patologias. Sabe-se que as instituições locais possuem suporte suficiente para a realização destes testes, não havendo a necessidade de enviar as amostras para um laboratório de apoio em outra cidade. Além disso, a correta orientação para trabalhadores em saúde e usuários também é imprescindível para o bom andamento do diagnóstico e estabelecimento de condutas terapêuticas. 


\section{Elaboração de questões}

A solução para o problema poderia advir da implementação de um fluxo de diagnóstico visando uma parceria entre Serviço de Assistência Especializada (SAE) e Centro de Testagem e Aconselhamento (CTA) para ISTs e uma instituição capaz de realizar a testagem, neste caso o Laboratório de Biologia Molecular da Universidade Franciscana. Essa parceria traria benefícios não somente para a população santa-mariense que tem interesse pelo diagnóstico, mas também para todos os alunos que fazem parte da instituição, resultando em um importante campo de práticas. Além do estabelecimento da parceria, também possuem impacto na qualificação dos processos a elaboração de um fluxograma de atendimento aos usuários em PrEP, um manual de coleta e diagnóstico de clamídia e gonorreia destinados aos profissionais de saúde, além da construção de folders informativos para a orientação dos usuários.

\section{Busca de novas informações}

Durante a reunião, foi informado sobre a falta da realização do diagnóstico de Chlamydia trachomatis e Neisseria gonorrohoreae. Com isso, passou-se a buscar informações sobre coleta e diagnóstico, na literatura já existente sobre o assunto, a fim de estabelecer um fluxograma que auxiliasse na implementação da detecção desses patógenos nas amostras enviadas pelo SAE/CTA ao Laboratório de Biologia Molecular da Universidade Franciscana.

\section{Construção de novos significados}

\section{a) Elaboração do fluxograma}

Após as etapas de problematização, foi estabelecido como prioridade para o Serviço de Assistência Especializada (SAE) e Centro de Testagem e Aconselhamento (CTA) a construção de um fluxograma para diagnóstico de Chlamydia trachomatis e Neisseria gonorrohoreae (Figura 1), uma vez que este não era realizado no centro de referência.

Segundo o fluxo estabelecido, o paciente, após ser indicado à PrEP, passa pela primeira consulta na qual serão solicitados exames como testagem para detecção de HIV, avaliação da função hepática e renal e testes para detecção de outras IST's. Dentre as IST's estão a sífilis e hepatites B e C, que já são solicitadas atualmente, clamídia e gonococo. A coleta dessas últimas será realizada por meio de swab retal e de orofaringe e, posteriormente, o material será enviado para o Laboratório de Biologia Molecular da Universidade Franciscana para realização de ensaio de proteína C reativa (PCR). Tais coletas serão feitas semestralmente ou quando houver suspeita clínica. 
Figura 1 - Fluxograma para diagnóstico de Chlamydia trachomatis e Neisseria gonorrohoreae

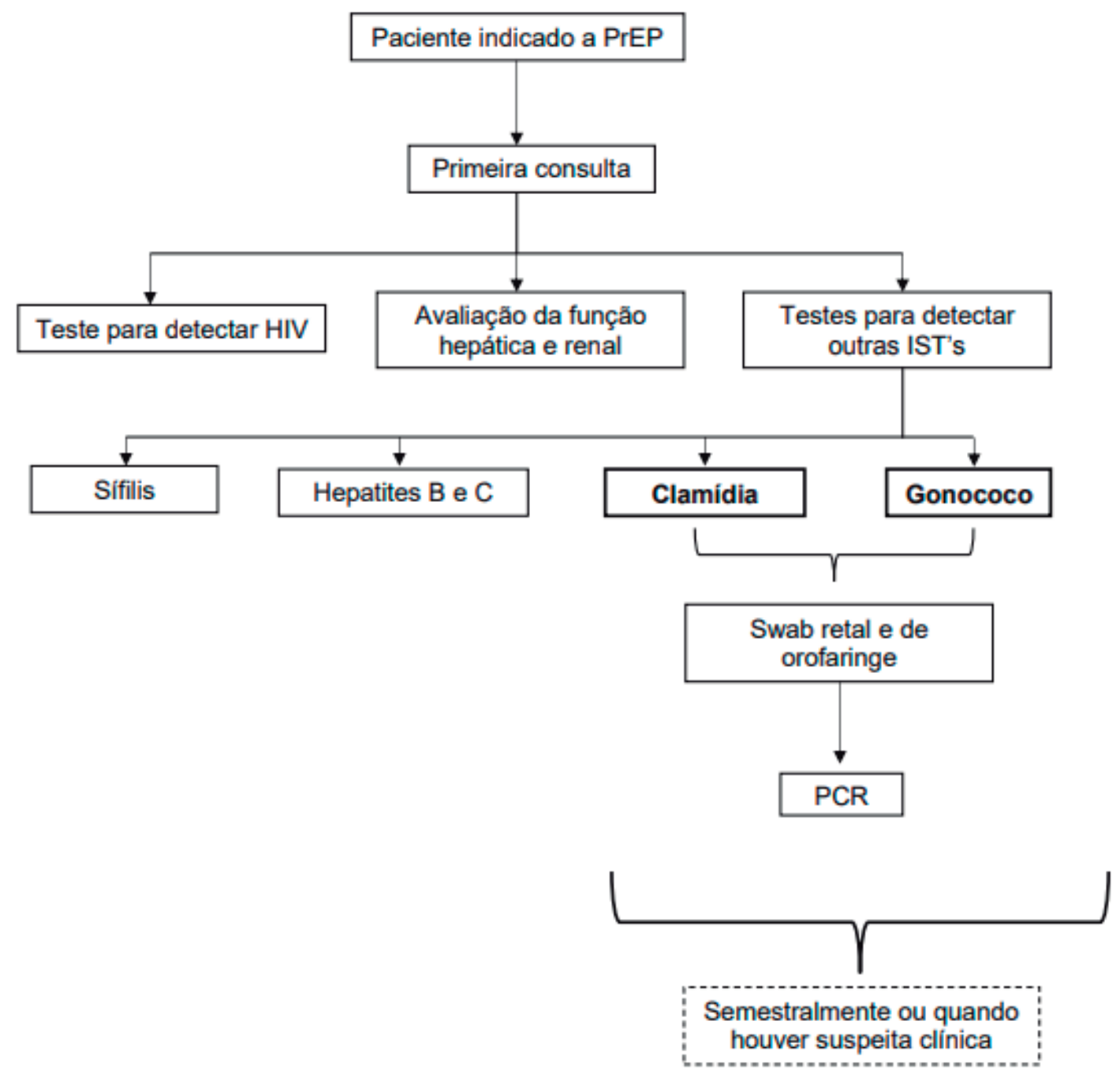

Fonte: elaboração própria.

\section{b) Elaboração do manual de procedimentos}

Após a análise transversal dos guidelines, protocolos e demais referências para padronização dos procedimentos, foi elaborado o "Manual de Coleta para Diagnóstico de Clamídia e Gonorreia". Compõe o texto esclarecimentos sobre as etapas para a coleta de material biológico; orientações ao usuário quanto a coleta e ao preparo; procedimentos para a coleta e armazenamento do material biológico; critérios de aceitabilidade no recebimento das amostras; orientações para o transporte e acesso aos resultados das análises.

A elaboração de protocolos e manuais de procedimentos pode ser uma ferramenta importante na linha de cuidado em Saúde Sexual e Reprodutiva, apoiando a qualificação da assistência e auxiliando na organização dos serviços, uma vez que consideram a atualidade do conhecimento científico e tecnológico e incluem informações a serem respeitados no processo assistencial. A padronizações das técnicas devem explicitar informações relativas à organização da oferta de ações de saúde neste sistema, em específico. 


\section{c) Elaboração dos materiais informativos}

A comunicação é uma ferramenta indispensável no contexto da assistência, sendo a forma escrita amplamente utilizada nas atividades educativas a fim de promover saúde, prevenir doenças, desenvolver habilidades e favorecer a autonomia do paciente. O material educativo impresso representa uma oportunidade importante para veicular mensagens de saúde e para se facilitar o processo ensino-aprendizagem, já que permite consultas recorrentes. Existem algumas restrições ou limitações em seu uso, em função do linguajar utilizado pelos elaboradores e outras decorrentes de dificuldades de leitura, em decorrência da inadequação do material, das características do leitor, principalmente de seu grau de escolaridade.

O material informativo elaborado durante a proposta teve por objetivo promover a adequada coleta domiciliar de urina e coleta por swab anal. No primeiro caso, foram pontuadas as seguintes orientações: não ter urinado nas últimas 2 (duas) horas; não estar tomando antibióticos; se possível, manter abstinência sexual por 3 dias; não estar menstruada; não estar utilizando creme vaginal, além de orientações para a coleta. No caso do $s w a b$ anal, foram reforçadas orientações sobre a observância do tempo decorrido desde a última evacuação, conduta apropriada em presença de lesões anais e uso de antibióticos, além da orientação para a coleta em si.

\section{Avaliação dos processos e produtos}

A efetiva implementação do fluxograma e das atividades de educação em saúde contempla o desenvolvimento de uma série de ações que venham a contribuir para a redução da morbimortalidade relacionada às ISTs. Em mulheres grávidas as infecções causam riscos não só para a mãe como também para o bebê, pois podem levar a vários desfechos adversos, incluindo ruptura prematura de membranas e partos prematuros (RODRIGUES; OLIVEIRA; GARCIA, 2018). No contexto da saúde da gestante, pode-se identificar a demanda reprimida e ampliar a oferta de teste de triagem para identificação de infecção por Chlamydia trachomatis e Neisseria gonorrohoreae, e por consequência, realizar tratamento farmacológico adequado.

Outra possibilidade consiste na ampliação do acesso aos testes para parceiros, sendo este o processo pelo qual os contatos sexuais de um paciente-índice (aquele que recebeu um diagnóstico de IST) são identificados e informados de sua exposição e convidados a realizar testes, aconselhamento e, se necessário, tratamento. Esta testagem deve ocorrer de forma voluntária dentro de ambientes propícios, sociais e legais (CAVALCANTE et al., 2016).

Por fim, a identificação dos casos de infecção por Chlamydia trachomatis e Neisseria gonorrohoreae pode nortear a promoção de ações educativas, por meio de campanhas de saúde, intervenções relacionadas ao uso de preservativos, prevenção de outras IST e gravidez na adolescência. A efetiva interação entre profissional da saúde e usuários é uma ferramenta importante 
para prevenir doenças e promover saúde, bem como orientar rotinas direcionadas ao autocuidado (GONÇALVES et al., 2020).

\section{CONCLUSÃO}

O trabalho realizado nesta atividade de extensão representou oportunidade de crescimento profissional e troca mútua entre docentes e discentes dos cursos de graduação e residência e técnicos municipais. A possibilidade do conhecimento da realidade desses municípios oportunizadas pela disciplina extensionista levou os estudantes à constatação do papel do SUS para a implementação de ações da política de saúde sexual e reprodutiva e do seu impacto na melhora na qualidade de vida dos cidadãos, assim como a constatação de que há ainda muito a ser feito.

A elaboração do protocolo, do manual e dos materiais informativos contou com a participação dos diversos atores, com opiniões por vezes divergentes, e representou um processo rico em trocas de conhecimentos e experiências. No trabalho realizado, ficou evidente a importância da observação de aspectos técnicos, éticos e políticos durante o processo de elaboração dos materiais. Destaca-se que a visão ampliada e integral da situação favorece o estabelecimento de relações mais cooperativas e solidárias entre as instituições envolvidas para responder às demandas crescentes dos cidadãos por serviços de saúde, de forma mais resolutiva, acessível e de melhor qualidade. Além disso, a oportunidade de formação universitária no contexto do SUS representa um importante passo para a qualificação dos serviços de saúde.

\section{REFERÊNCIAS}

BEZERRA, Vladimir. Cenários políticos brasileiros, conquistas e desafios para as políticas públicas de saúde no contexto da prevenção e tratamento do HIV/AIDS e IST’s. O Social em Questão, v. 22, n. 45, p. $13-34,2019$.

BRASIL. Ministério da Saúde. Assistência Integral à Saúde da Mulher: Bases de Ação Programática. Brasília (DF): Ministério da Saúde; 1985.

BRASIL. Lei n. 9.263 de 12 de janeiro de 1996. Regula o $\S 7^{\circ}$ do art. 226 da Constituição Federal, que trata do planejamento familiar, estabelece penalidades e dá outras providências. Diário Oficial da União, Brasília (DF), 15 jan. 1996.

BRASIL. Ministério da Saúde. Secretaria de Atenção à Saúde. Departamento de Ações Programáticas Estratégicas. Política nacional de atenção integral a saúde do homem - princípios e diretrizes. Brasília (DF): Ministério da Saúde; 2008. 
BRASIL. Ministério da Saúde. Instituto Sírio-Libanês de Ensino e Pesquisa. Protocolos da Atenção

Básica: Saúde das Mulheres. Brasília (DF): Ministério da Saúde; 2016.

BRASIL. Ministério da Saúde. Saúde Sexual e Saúde Reprodutiva: os homens como sujeitos de cuidado. Brasília (DF): Ministério da Saúde; 2018.

CAVALCANTE, Elani Graça Ferreira. G. F.; MIRANDA, Mahara Coelho Crisostomo; CARVALHO, Ana Zaiz Flores Hormain Teixeira de. Notificação de parceiros sexuais com infecção sexualmente transmissível e percepções dos notificados. Revista da Escola de Enfermagem da USP, v. 50, n. 3, p. $450-457,2016$.

CORRÊA, Sonia; ALVES, José Eustáquio Diniz; JANNUZZI, Paulo de Martino. Direitos e saúde sexual e reprodutiva: marco teórico-conceitual e sistema de indicadores. In: CAVENAGHI, Suzana, Indicadores municipais de saúde sexual e reprodutiva, Brasília: UNFPA, p. 27-62, 2015.

DIAS, Mariana de Sales et al. Síntese de evidências para políticas de saúde: enfrentamento da sífilis congênita no âmbito da atenção primária à saúde. BIS. Boletim do Instituto de Saúde, v. 20, n. 2, p. 89, 2019.

FELISBINO-MENDES, Mariana Santos et al. Análise dos indicadores de saúde sexual e reprodutiva de adolescentes brasileiros, 2009, 2012 e 2015. Revista Brasileira de Epidemiologia, v. 21, p. e180013, 2018.

FONSECA, Angélica. Prevenção às DST/aids no ambiente escolar. Interface (Botucatu). n. 6, v. 11, p. 71-88, 2002.

FORPROEX. Indissociabilidade ensino-pesquisa-extensão e a flexibilização curricular: uma visão da extensão / Fórum de Pró-Reitores de Extensão das Universidades Públicas Brasileiras. Porto Alegre: UFRGS; Brasília: MEC/SESu, 2006. Disponível em: https://bit.ly/3aROdP5. Acesso em: 26 jul. 2021.

GALVÃO, Taís Freire; COSTA, Carlos Henrique Nery; GARCIA, Leila Posenato. Atenção integral às Pessoas com Infecções Sexualmente Transmissíveis. 2021.

GIOVANNI, Geraldo di; NOGUEIRA, Marco Aurélio. Dicionário de políticas públicas. 3. ed., São Paulo: Editora UNESP, 2018. 
GONÇALVES, Romário de Sousa et al. Educação em saúde como estratégia de prevenção e promoção da saúde de uma unidade básica de saúde. Brazilian Journal of Health Review, v. 3, n. 3, p. 5811-5817, 2020.

HIGA, Elza de Fátima Ribeiro et al. A intersetorialidade como estratégia para promoção da saúde sexual e reprodutiva dos adolescentes. Interface-Comunicação, Saúde, Educação, v. 19, p. 879-891, 2015.

LEMOS Adriana. Direitos sexuais e reprodutivos: percepção dos profissionais da atenção primária à saúde. Saúde Debate. n. 38, v. 101, p. 244-253, 2014.

LIMA, Valéria Vernaschi. Espiral construtivista: uma metodologia ativa de ensino-aprendizagem. Interface (Botucatu), Botucatu, v. 21, n. 61, p. 421-434, June 2017.

MACHADO, Cristiani Vieira; LIMA, Luciana Dias de; BAPTISTA, Tatiana Wargas de Faria. Políticas de saúde no Brasil em tempos contraditórios: caminhos e tropeços na construção de um sistema universal. Cadernos de Saúde Pública, v. 33, p. e00129616, 2017.

PAIVA, Carla Cardi Nepomuceno de; CAETANO, Rosângela. Avaliação de implantação das ações de saúde sexual e reprodutiva na Atenção Primária: revisão de escopo. Escola Anna Nery, v. 24, n. 1, 2020.

RODRIGUES, Camila Aparecida Coelho; DE OLIVEIRA, Laryssa; GARCIA, Patrícia Guedes. Prevalência de Infecções por Neisseria Gonorrhoeae em Gestantes: Uma Revisão Sistemática. Revista Brasileira de Ciências Médicas e da Saúde, v. 6, n. 6, p. 1-5, 2018.

TELO, Shana Vieira; WITT, Regina Rigatto. Saúde sexual e reprodutiva: competências da equipe na Atenção Primária à Saúde. Ciência \& Saúde Coletiva, v. 23, p. 3481-3490, 2018. 